\title{
A Signal-Centric Perspective on the Evolution of Symbolic Communication (Supplementary Material)
}

\author{
QUINTINO FRANCESCO LOTITO, University of Trento \\ LEONARDO LUCIO CUSTODE, University of Trento \\ GIOVANNI IACCA, University of Trento
}

\section{CONSTELLATIONS FOR THE SIGNALING SYSTEMS EVOLVED AT VARIOUS LEVELS OF NOISE}

In Figures 2-8 we show the constellations of signals of the best signaling systems evolved in each of the 20 evolutionary runs in a subset of the experiments with noise reported in Section 5.3 of the main text, namely: regression and classification settings, with unlimited amplitude, 3 trials per concept, and Gaussian noise $\mathcal{N} \sim$ $\left(0, \sigma^{2}\right)$, with $\sigma=0.1, \sigma=0.2, \sigma=0.5$ and $\sigma=1$. 


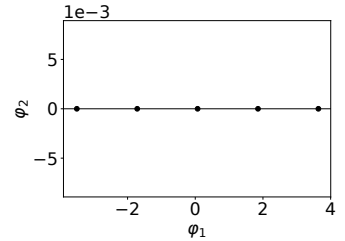

Run 1

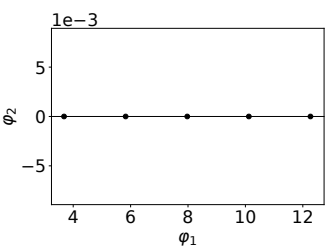

Run 5

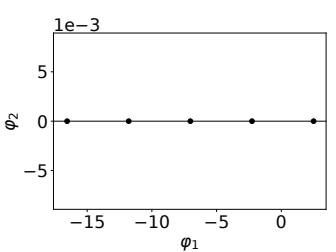

Run 9

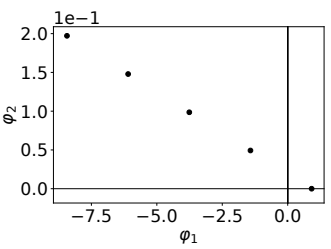

Run 13

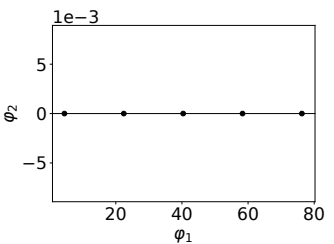

Run 17

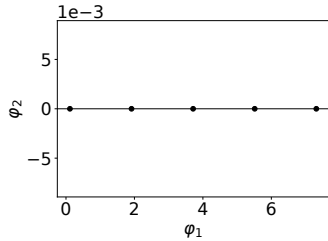

Run 2

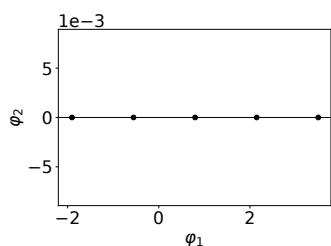

Run 6

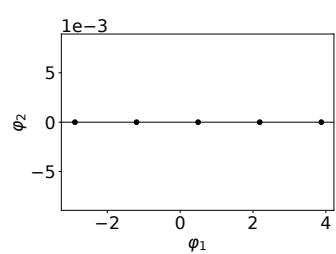

Run 10

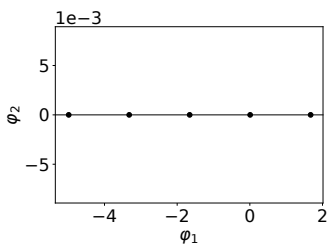

Run 14

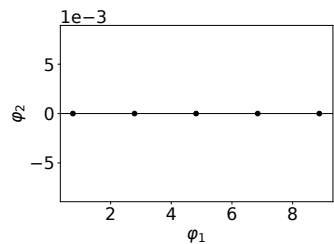

Run 18

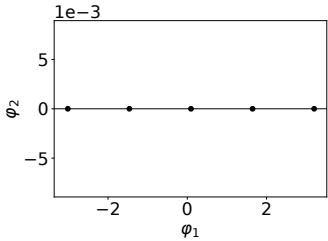

Run 3

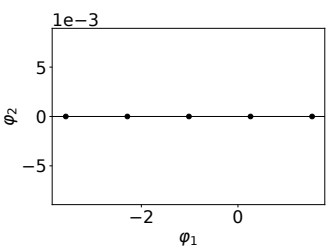

Run 7

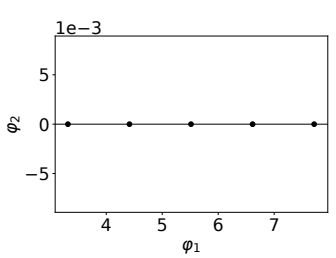

Run 11

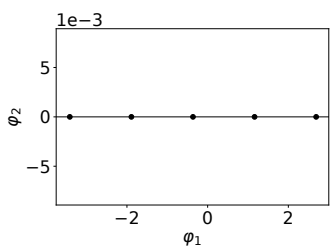

Run 15

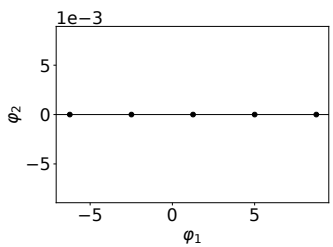

Run 19

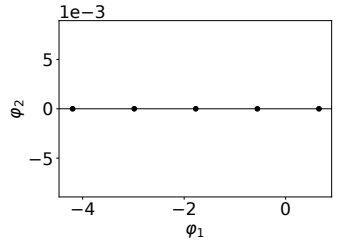

Run 4

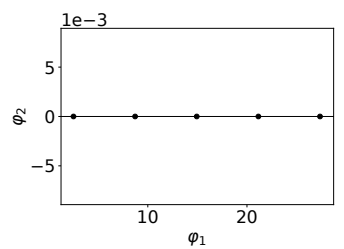

Run 8

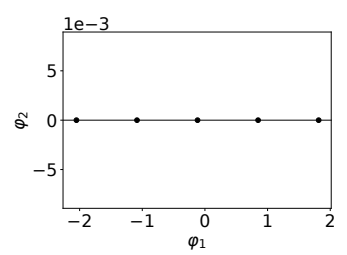

Run 12

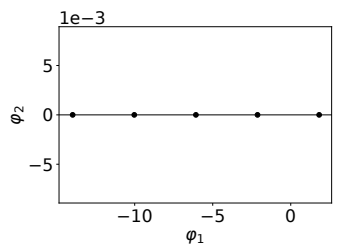

Run 16

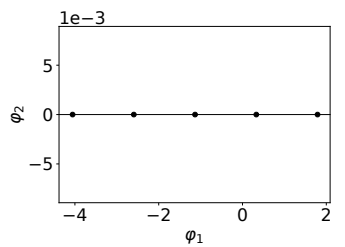

Run 20

Fig. 1. Constellation of signals of the best signaling systems evolved in the regression setting, unlimited amplitude, 3 trials, $\sigma=0.1$. 


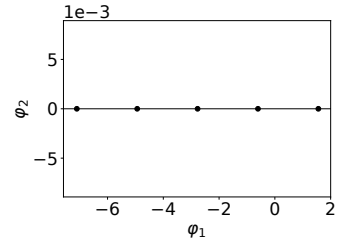

Run 1

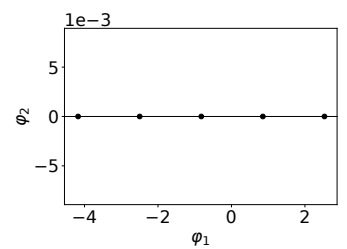

Run 5

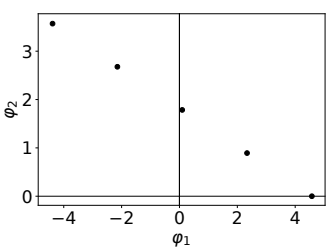

Run 9

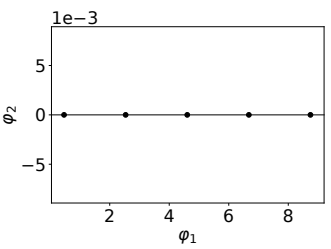

Run 13

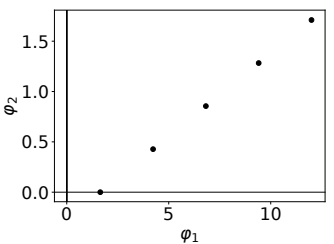

Run 17

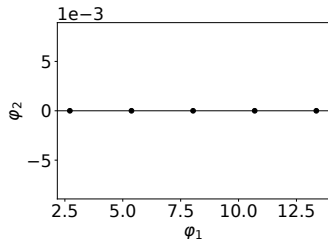

Run 2

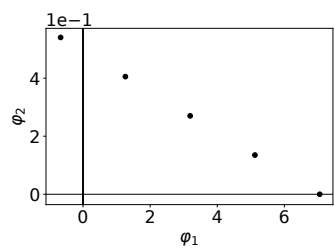

Run 6

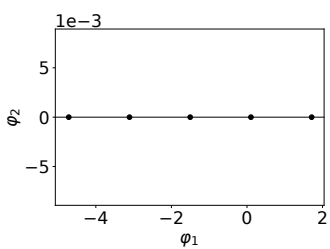

Run 10

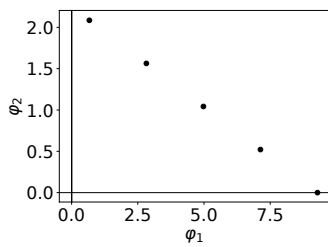

Run 14

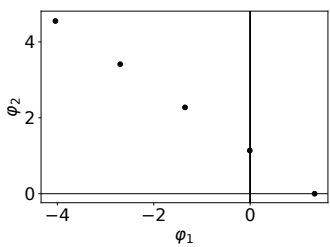

Run 18

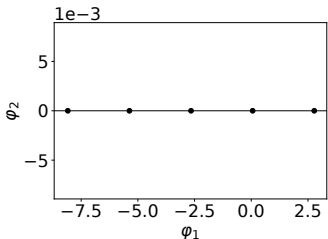

Run 3

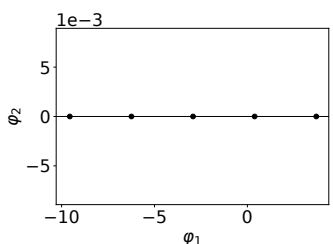

Run 7

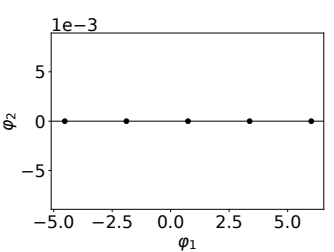

Run 11

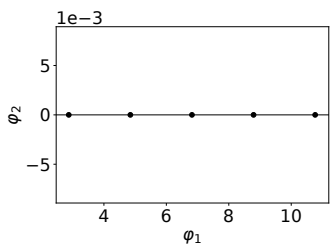

Run 15

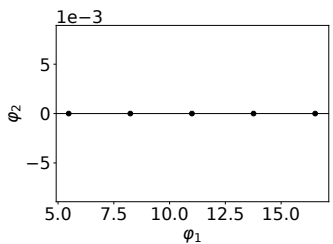

Run 19

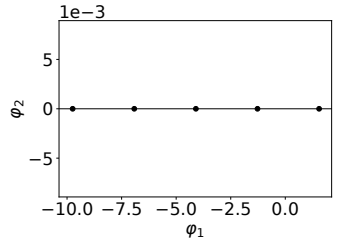

Run 4

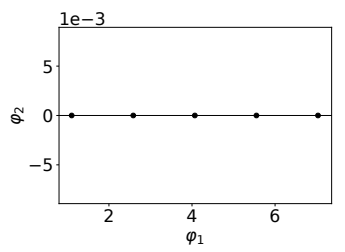

Run 8

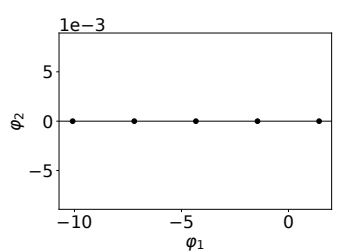

Run 12

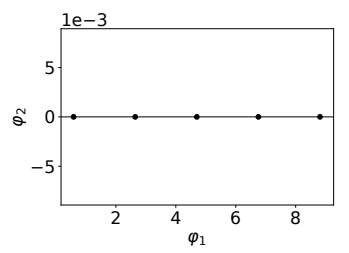

Run 16

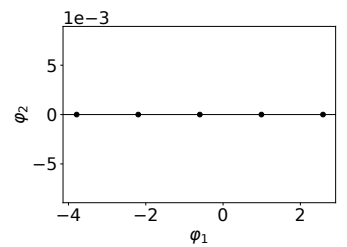

Run 20

Fig. 2. Constellation of signals of the best signaling systems evolved in the regression setting, unlimited amplitude, 3 trials, $\sigma=0.2$. 


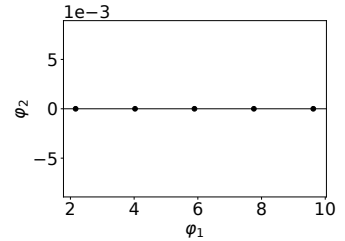

Run 1

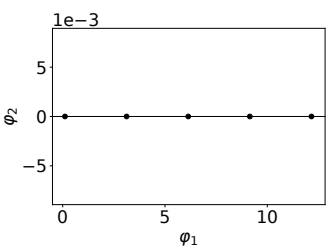

Run 5

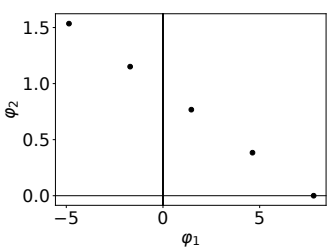

Run 9

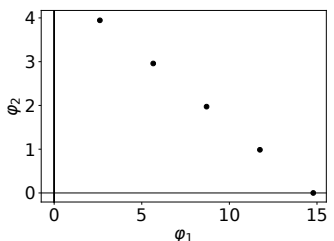

Run 13

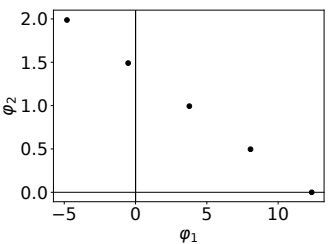

Run 17

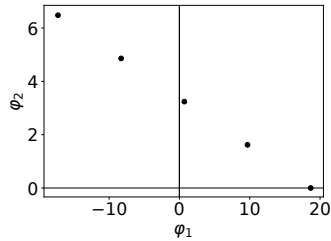

Run 2

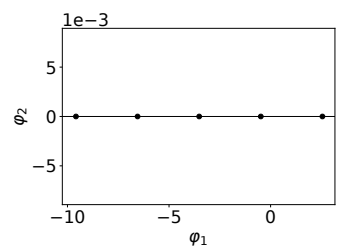

Run 6

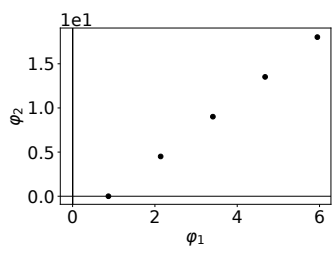

Run 10

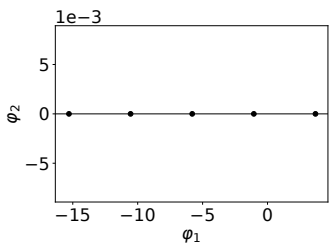

Run 14

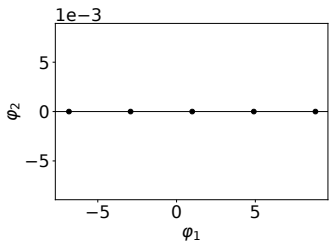

Run 18

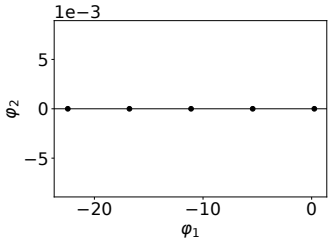

Run 3

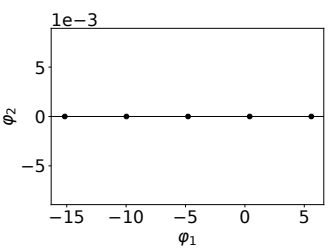

Run 7

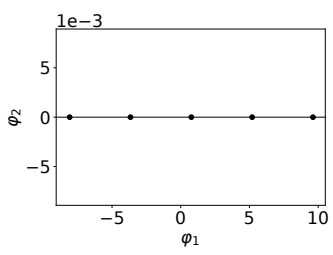

Run 11

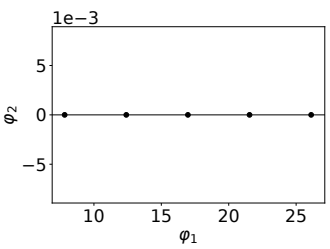

Run 15

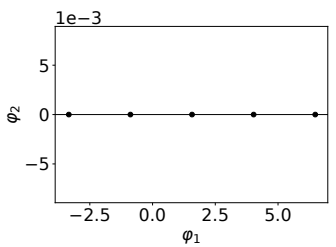

Run 19

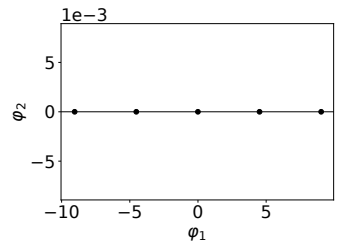

Run 4

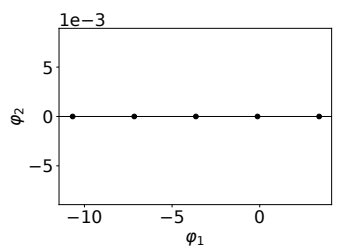

Run 8

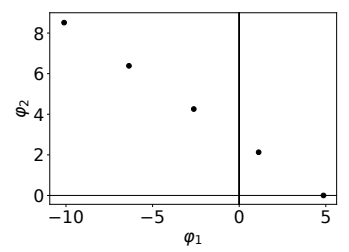

Run 12

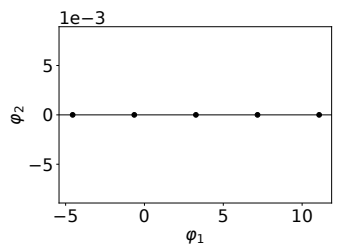

Run 16

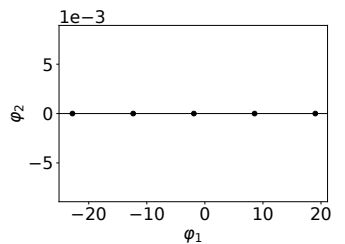

Run 20

Fig. 3. Constellation of signals of the best signaling systems evolved in the regression setting, unlimited amplitude, 3 trials, $\sigma=0.5$. 


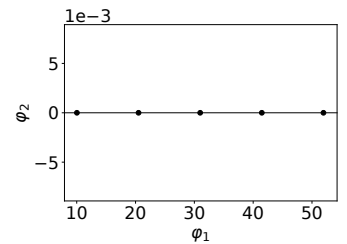

Run 1

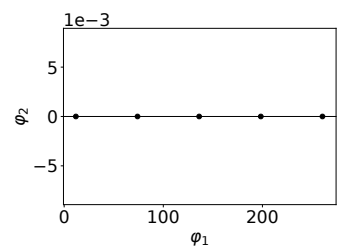

Run 5

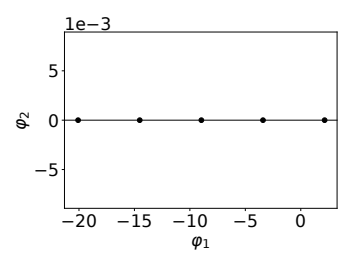

Run 9

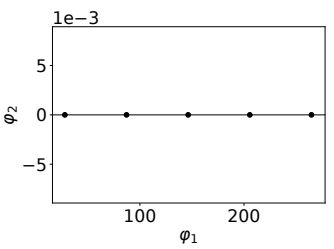

Run 13

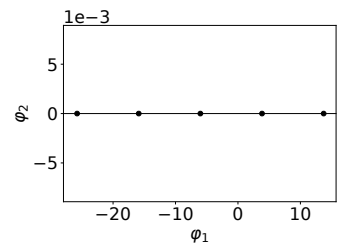

Run 17

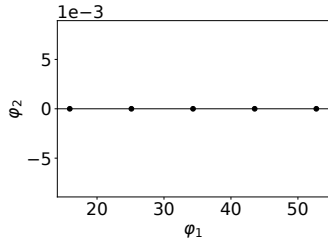

Run 2

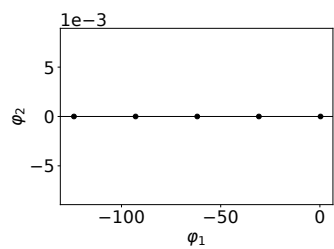

Run 6

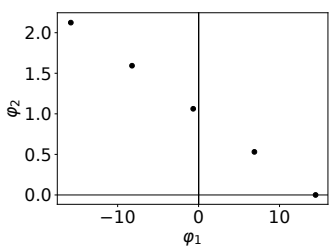

Run 10

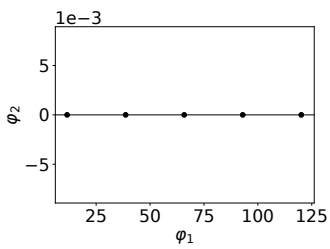

Run 14

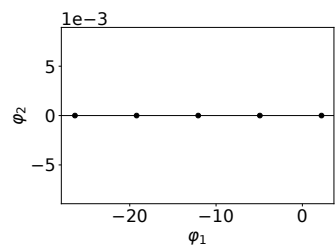

Run 18

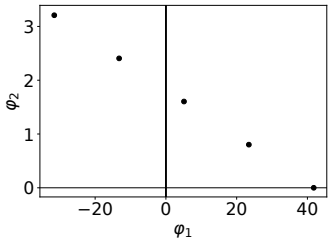

Run 3

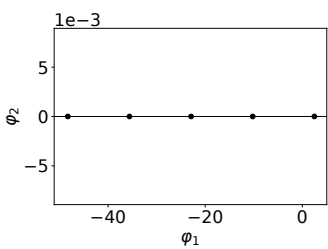

Run 7

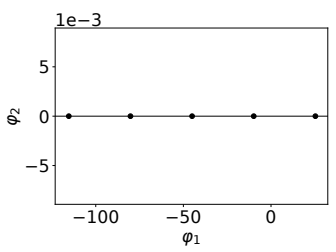

Run 11

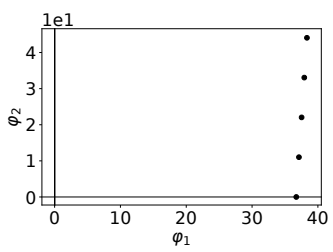

Run 15

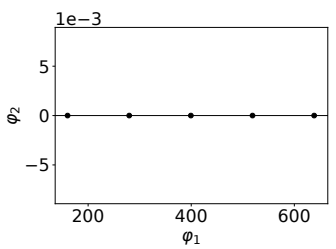

Run 19

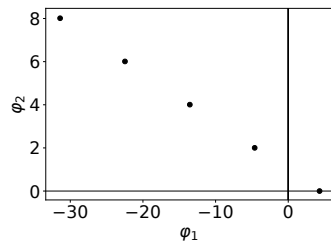

Run 4

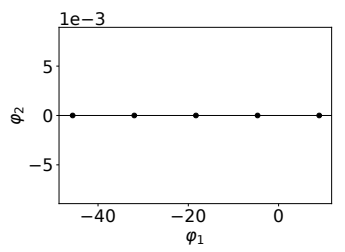

Run 8

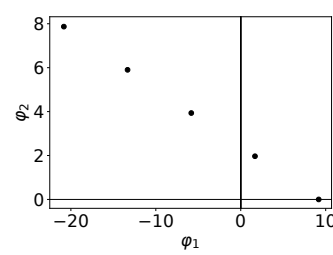

Run 12

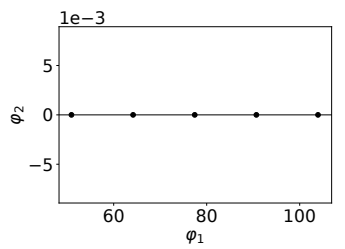

Run 16

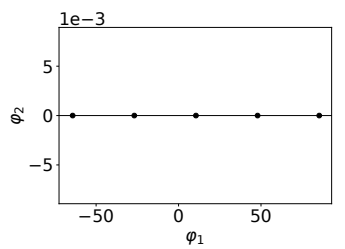

Run 20

Fig. 4. Constellation of signals of the best signaling systems evolved in the regression setting, unlimited amplitude, 3 trials, $\sigma=1$. 


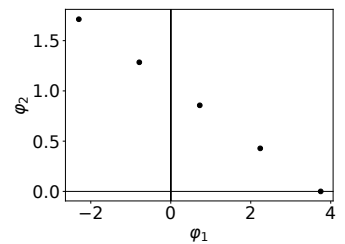

Run 1

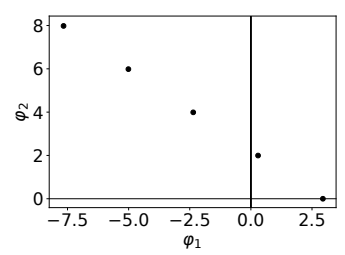

Run 5

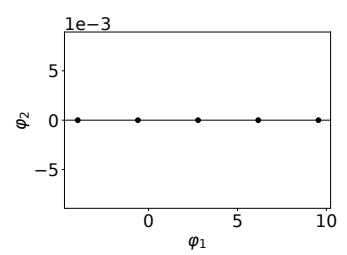

Run 9

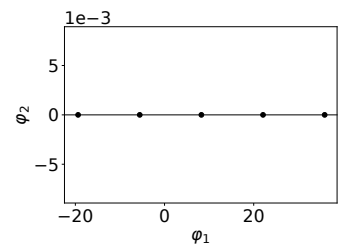

Run 13

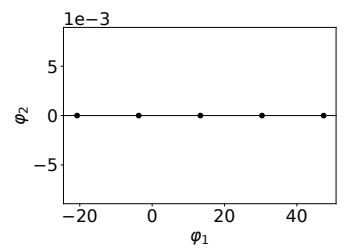

Run 17

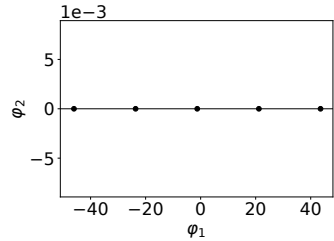

Run 2

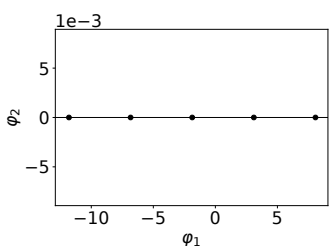

Run 6

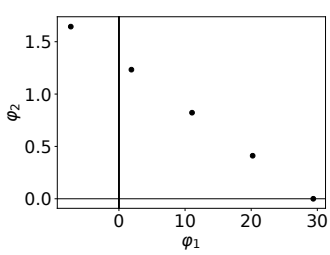

Run 10

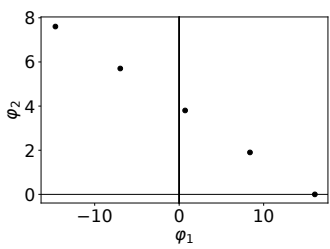

Run 14

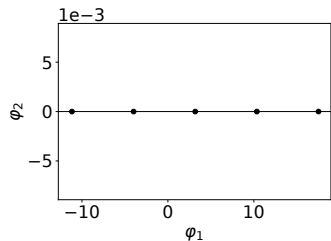

Run 18

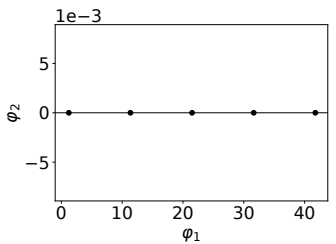

Run 3

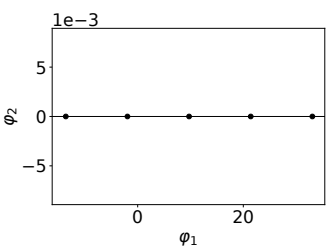

Run 7

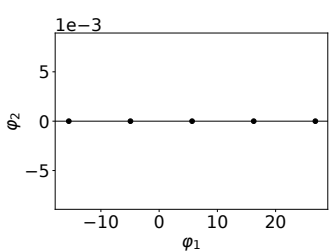

Run 11

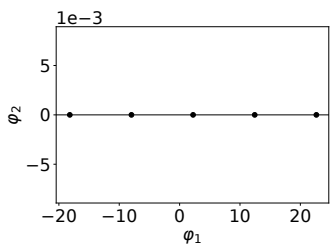

Run 15

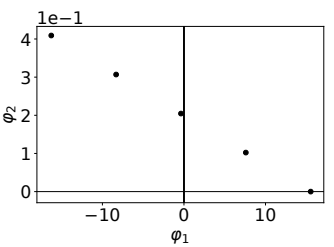

Run 19

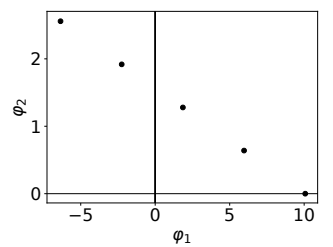

Run 4

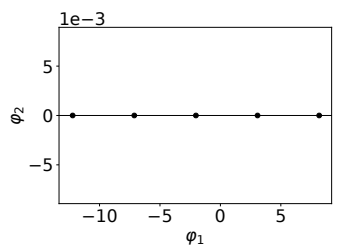

Run 8

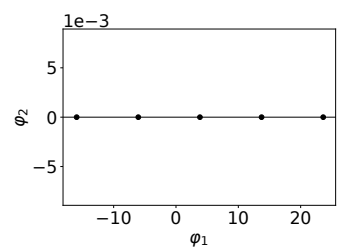

Run 12

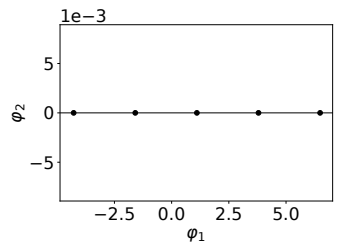

Run 16

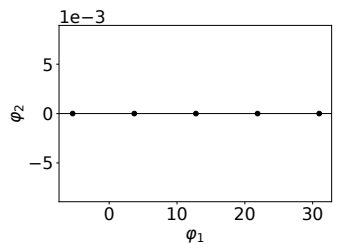

Run 20

Fig. 5. Constellation of signals of the best signaling systems evolved in the classification setting, unlimited amplitude, 3 trials, $\sigma=0.1$. 


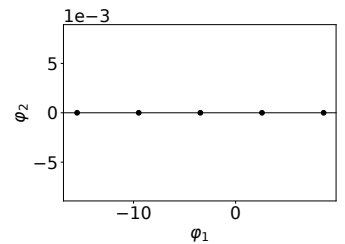

Run 1

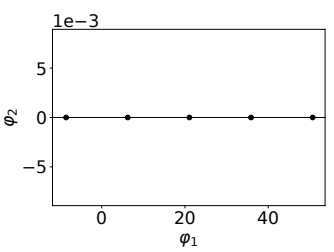

Run 5

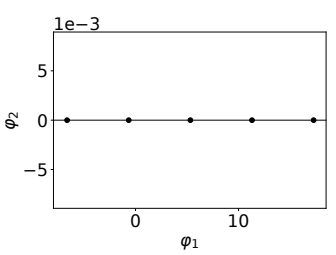

Run 9

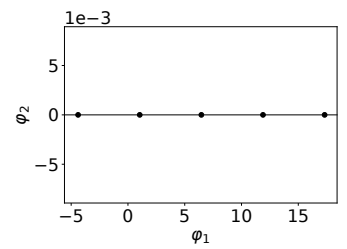

Run 13

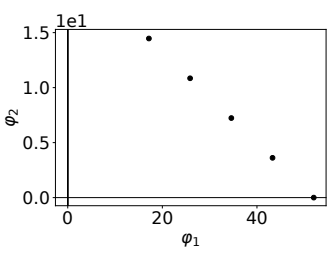

Run 17

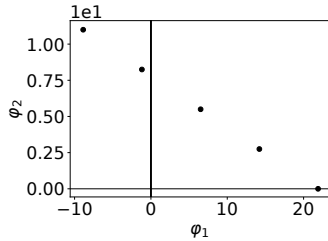

Run 2

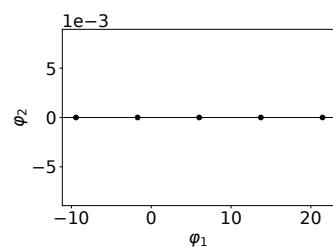

Run 6

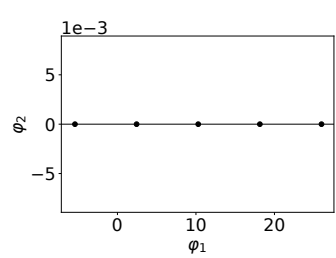

Run 10

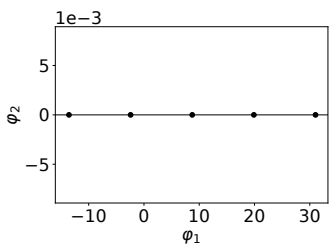

Run 14

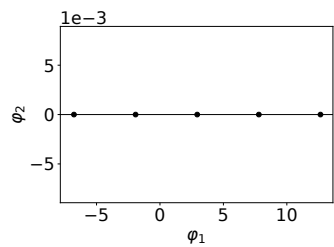

Run 18

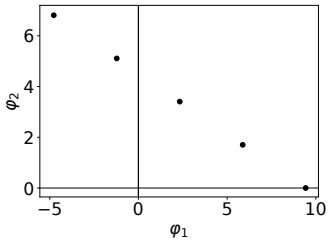

Run 3

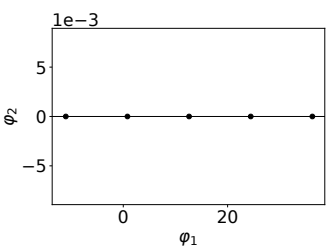

Run 7

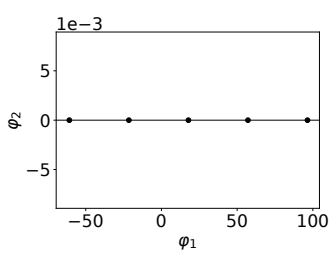

Run 11

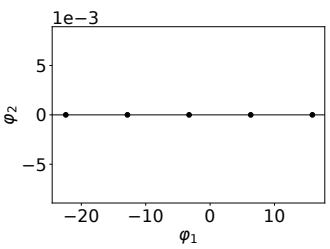

Run 15

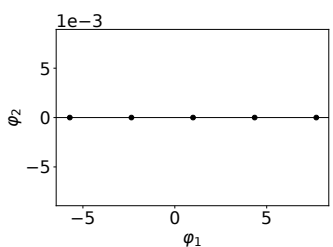

Run 19

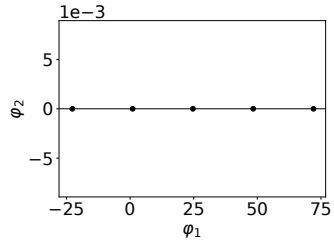

Run 4

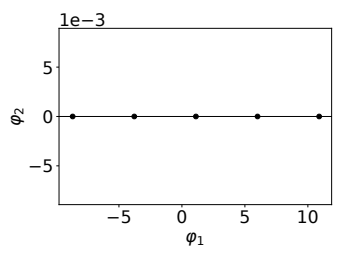

Run 8

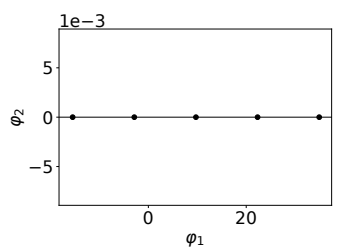

Run 12

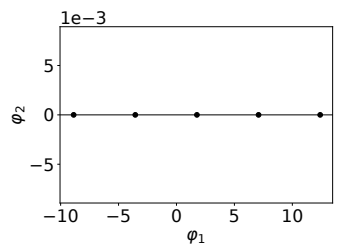

Run 16

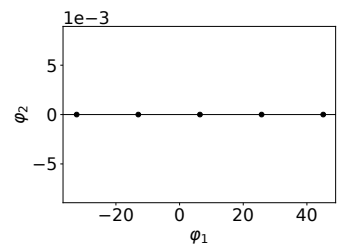

Run 20

Fig. 6. Constellation of signals of the best signaling systems evolved in the classification setting, unlimited amplitude, 3 trials, $\sigma=0.2$. 


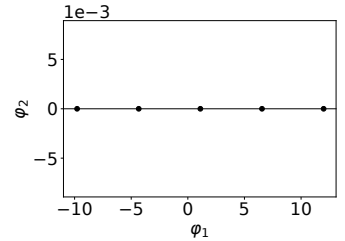

Run 1

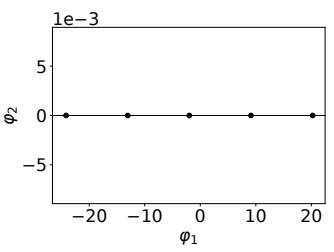

Run 5

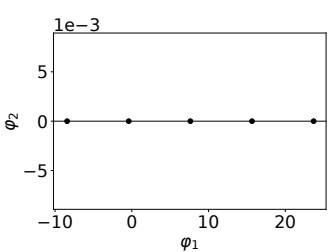

Run 9

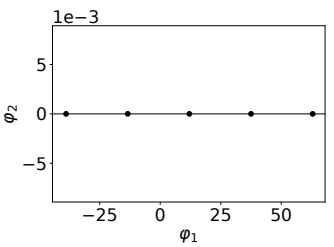

Run 13

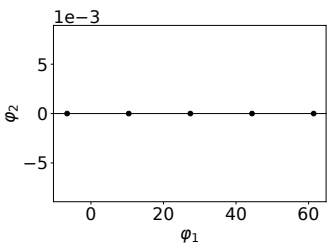

Run 17

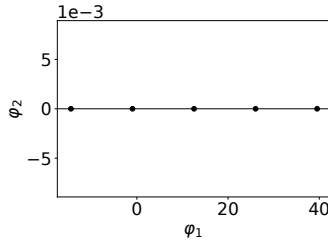

Run 2

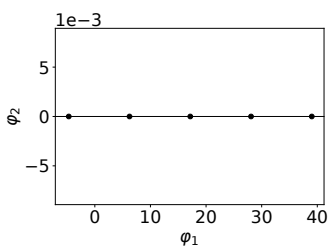

Run 6

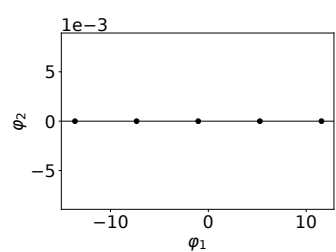

Run 10

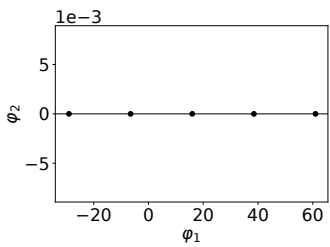

Run 14

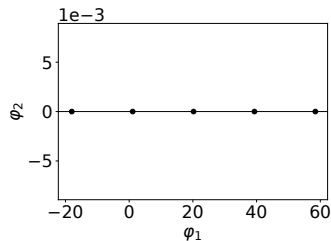

Run 18

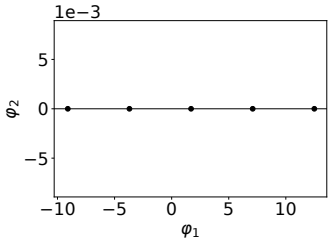

Run 3

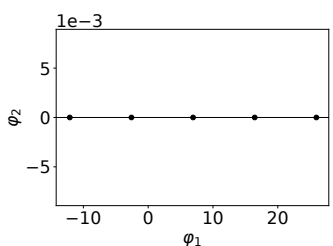

Run 7

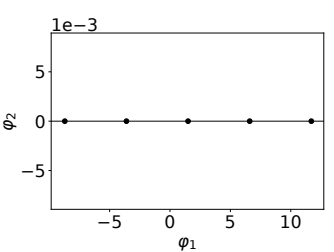

Run 11

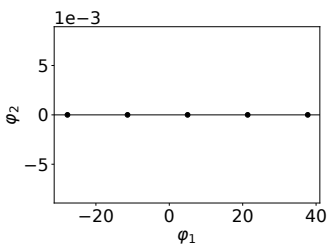

Run 15

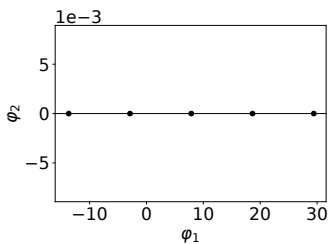

Run 19

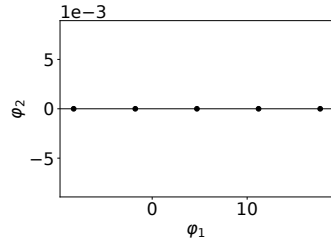

Run 4

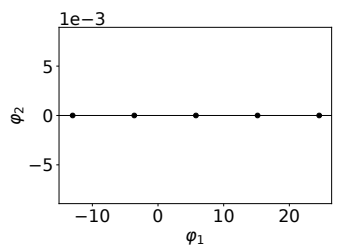

Run 8

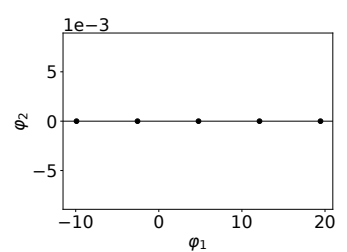

Run 12

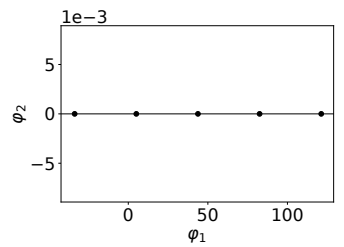

Run 16

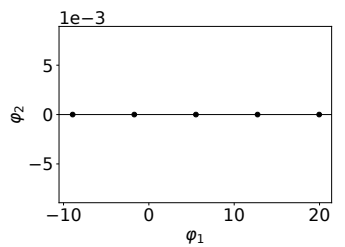

Run 20

Fig. 7. Constellation of signals of the best signaling systems evolved in the classification setting, unlimited amplitude, 3 trials, $\sigma=0.5$. 


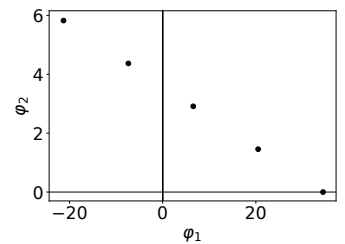

Run 1

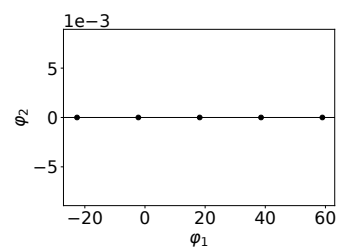

Run 5

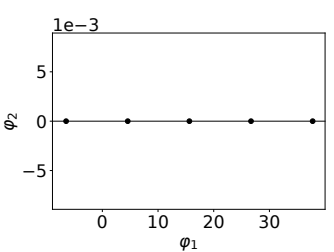

Run 9

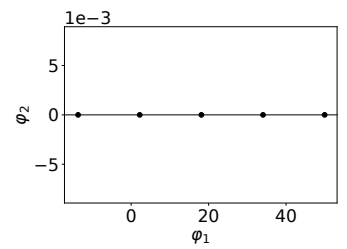

Run 13

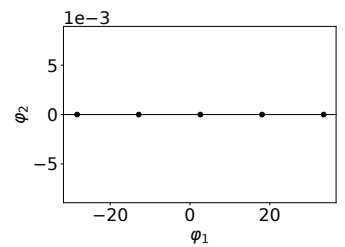

Run 17

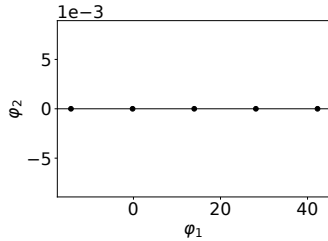

Run 2

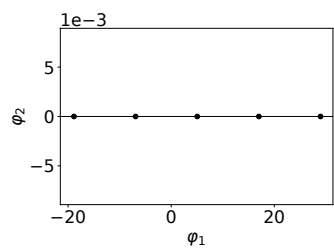

Run 6

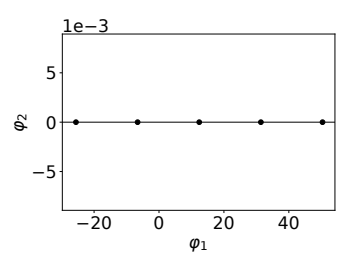

Run 10

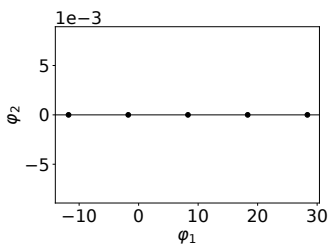

Run 14

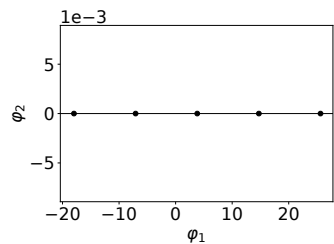

Run 18

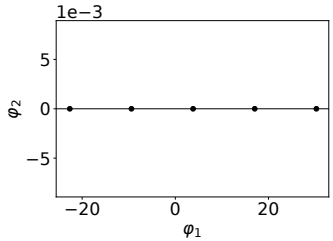

Run 3

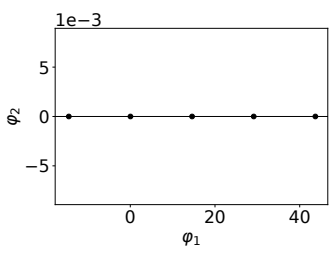

Run 7

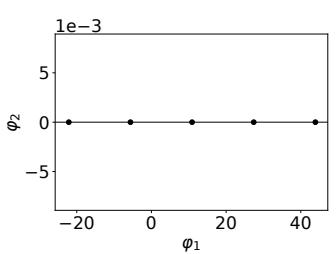

Run 11

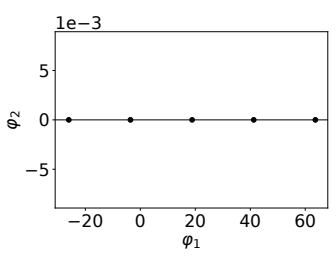

Run 15

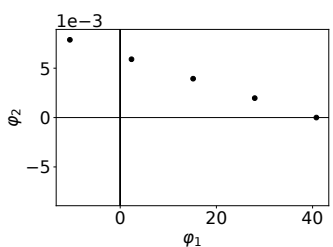

Run 19

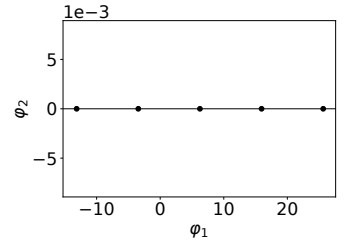

Run 4

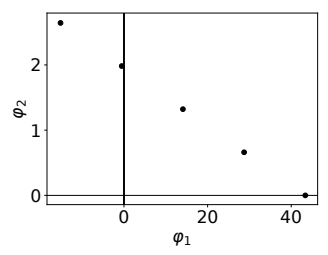

Run 8

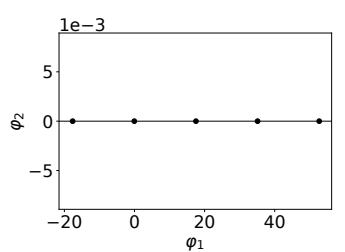

Run 12

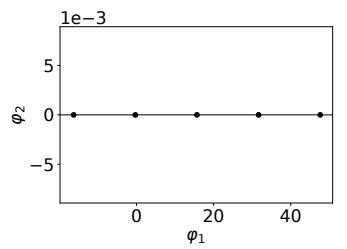

Run 16

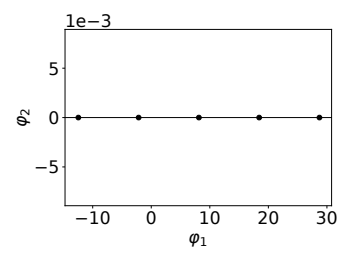

Run 20

Fig. 8. Constellation of signals of the best signaling systems evolved in the classification setting, unlimited amplitude, 3 trials, $\sigma=1$. 


\section{EXAMPLES OF EVOLVED SENDER/RECEIVER NETWORKS}

In Figures 9-10 we show some examples of the networks evolved in the regression and classification settings, respectively, with both unlimited and limited amplitude, 3 trials per concept, and Gaussian noise $\mathcal{N} \sim\left(0, \sigma^{2}\right)$, with $\sigma=0.1$.

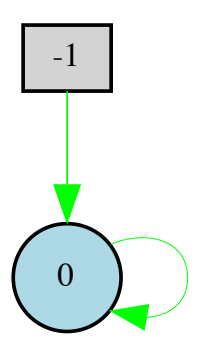

(a) Regression, unlimited: sender

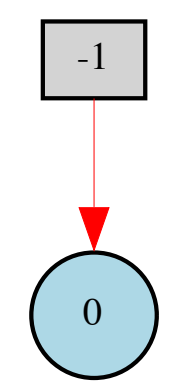

(c) Regression, limited: sender

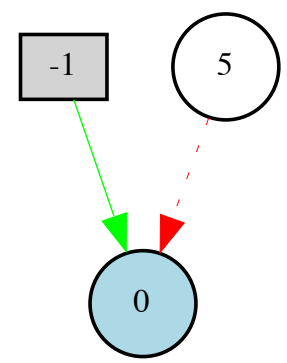

(b) Regression, unlimited: receiver

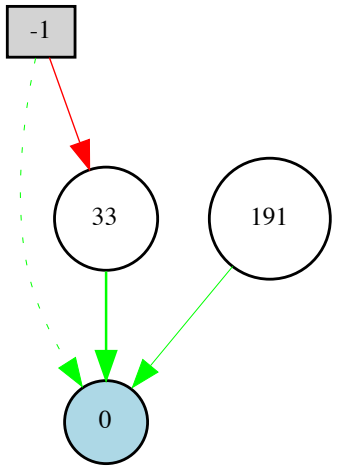

(d) Regression, limited: receiver

Fig. 9. Examples of the networks evolved in the regression settings, 3 trials, $\sigma=0.1$. 

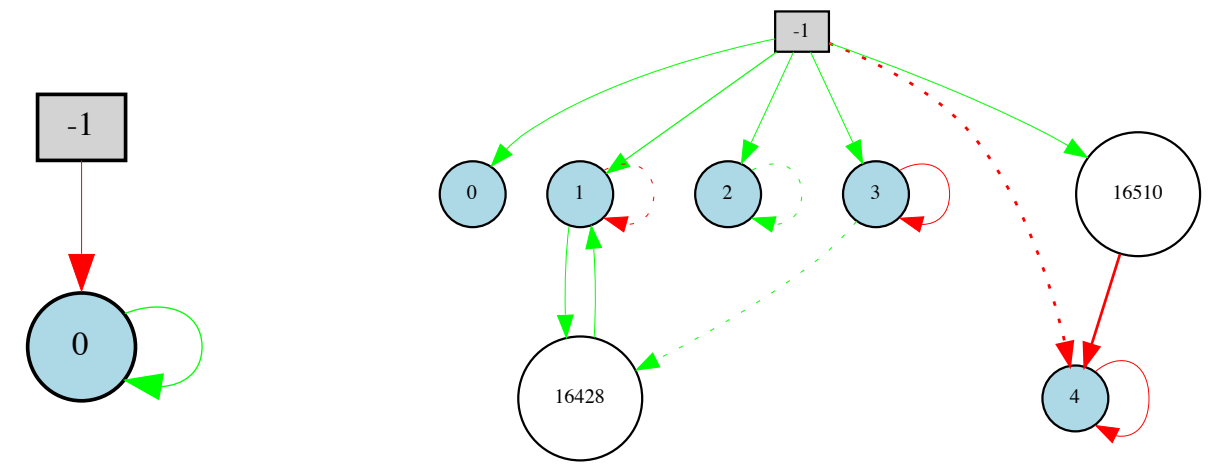

(a) Classification, unlimited: sender

(b) Classification, unlimited: receiver
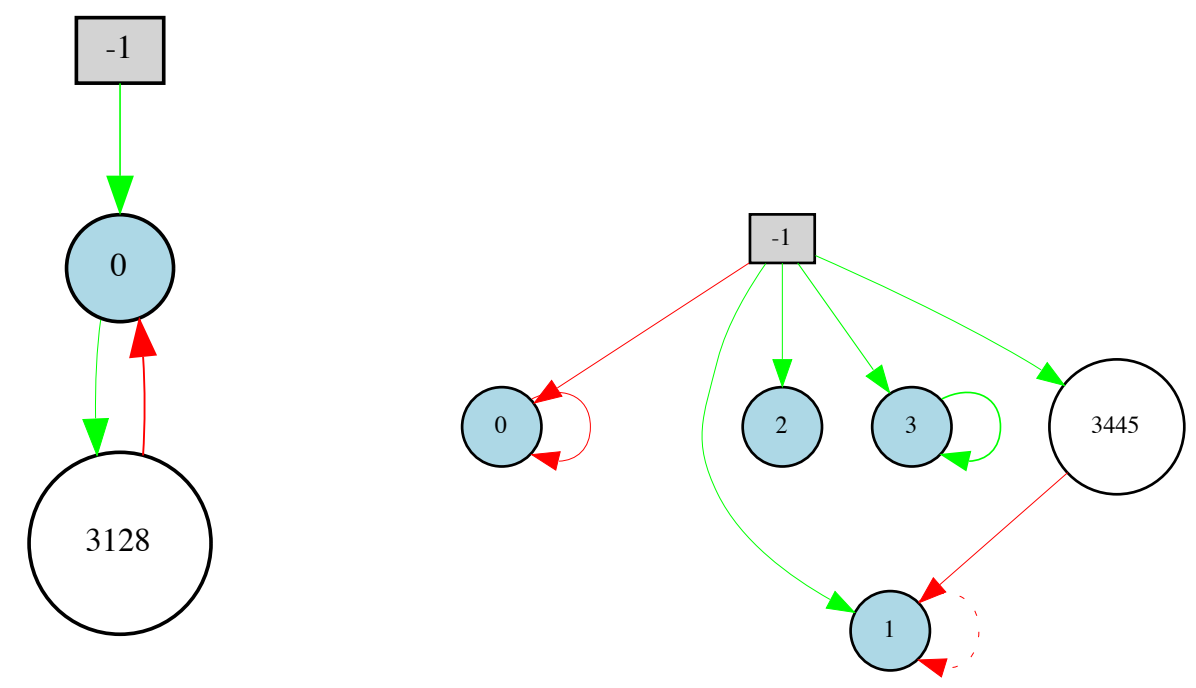

(c) Classification, limited: sender

(d) Classification, limited: receiver

Fig. 10. Examples of the networks evolved in the classification settings, 3 trials, $\sigma=0.1$. 